\title{
ORAL ULCERS- A CLINICOPATHOLOGICAL PROFILE...
}

\author{
Manjunath $K^{1}$, Smitha V. Manjunath ${ }^{2}$
}

${ }^{1}$ Assistant Professor, Department of ENT, Basaveshwara Medical College and Hospital \& Research Centre, Chitradurga. 2Postgraduate Student, Department of Oral Pathology \& Microbiology, Bapuji Dental College and Hospital, Davangere.

\section{ABSTRACT}

\section{BACKGROUND}

Oral ulcers have been an everyday problem and routinely patients with oral ulcers are treated symptomatically without even coming to a definitive conclusion of their own disease.

The objectives of this study are to identify various aetiological factors in ulcers of oral cavity and to study the benign and malignant nature of these lesions and outcome of its management.

\section{MATERIALS AND METHODS}

Study included patients attending ENT Outpatient Department for 12 months at our Hospital, wherein 68 cases were studied.

\section{RESULTS}

Out of 68 cases, 55 cases (80.8\%) were non-malignant ulcers and 13 cases (19.1\%) were malignant (including 3 cases of Leukoplakia). There were proper 10 cases of malignant ulcers (14.7\%), M: F : 4: 6, commonest age group between $3^{\text {rd }}$ to $4^{\text {th }}$ decade. Histopathologically, all the malignant ulcers were Squamous Cell Carcinoma.

\section{CONCLUSION}

Oral ulcers are common disease for which patient seeks medical advice. Clinical examination and histopathological examinations are mandatory investigations done for early diagnosis and treatment.

\section{KEYWORDS}

Histopathological Examination, Tobacco Chewers, Smoking, Betel Quid Chewers, Oral Cavity Lesions, Pre-Malignant Lesions (Leukoplakia), Malignant Lesions, Wedge Biopsy, Cancer Screening.

HOW TO CITE THIS ARTICLE: Manjunath K, Manjunath SV. Oral ulcers- a clinicopathological profile... J. Evolution Med. Dent. Sci. 2017;6(69):4938-4941, DOI: 10.14260/Jemds/2017/1071

\section{BACKGROUND}

Oral ulcers have been an everyday clinical condition to many otolaryngologists, physicians and dental surgeons. Many a times, patients with oral ulcers are treated symptomatically without even coming to a definitive conclusion of their malady. Among them many lesions are benign, pre-malignant and malignant with various aetiological factors.

Most of the oral cavity lesions are asymptomatic. The association between ill-fitting dentures and tobacco chewing, where most of them are inflammatory like aphthous ulcers, oral candidiasis, oral submucous fibrosis, mucoceles and nevus. No significant advancement in the treatment of oral lesions was found in recent years.

World Health Organisation (WHO) workshop end in 2005, terminology, definitions and classification of oral lesions with predisposing factors of malignant transformation has been discussed. The conditions considered as pre-malignant are leukoplakia, lichen planus, oral submucous fibrosis and erythroplakia. The pre-malignant conditions of the oral cavity are those which have potentiality to turn malignant in its due course if left untreated. In these treaties, tension was given to leukoplakia

Financial or Other, Competing Interest: None.

Submission 08-07-2017, Peer Review 03-08-2017,

Acceptance 08-08-2017, Published 28-08-2017.

Corresponding Author:

Dr. Smitha V. Manjunath,

Postgraduate Student,

Bapuji Dental College and Hospital, Davangere.

E-mail: eesha.1528@gmail.com

DOI: $10.14260 /$ jemds/2017/1071 and erythroplakia. Furthermore, lichen planus and oral submucous fibrosis are on rise. It was only in 1967 when an international reference centre for the study of oral precancerous lesion was established by the WHO. The incidence of oral cancer among patients who had tobacco smoking was 8.4-fold higher than that among patients who did not.

Membranous patch is an inflammatory condition, in which the cells of mucous membrane surface are eroded, an exudate is laid down on the surface and the whole necrotic layer is bound by fibrosis to the underlying tissue to form a false membrane classically seen in diphtheritic membrane.

Oral cavity ulcer may be an early sign of drug reaction or malignant transformation due to nutritional disorder and immunocompromised state. So, early diagnosis may help in better treatment and reduction of morbidity and mortality.

\section{Objectives of the Study}

- To identify clinical behaviour and various aetiological factors associated with ulcerative and membranous conditions in oral cavity and oropharynx.

- To know the significance in clinical morbidity and to determine the malignant potential in these lesions.

\section{MATERIALS AND METHODS}

- This study is a descriptive study from March 2016 to February 2017 for 12 months. The data was collected from the patients after taking history, detailed clinical examination and relevant investigations. 
- Clinical diagnosis was confirmed by throat swabs for culture, blood tests and biopsy for histopathological examination (HPE) wherever necessary. Patients were treated appropriately and regular followup of the cases was done.

Data was analysed by using descriptive statistics (mean, proportions, percentage) and presented in the form of tables, bar diagram, cone diagram and pie diagrams.

\section{RESULTS}

In our study of aetiopathology of ulcers and membranous lesions of oral cavity and oropharynx of the 68 cases, out of which 56 patients were above 40 years of age and 12 were below that age. P-value was 0.001 . Out of 68 , males were 46 cases $(67.6 \%)$ and females were 22 cases $(32.3 \%)$. The ratio of Male: Female was 2: 1 with male predominance.

\begin{tabular}{|c|c|c|c|}
\hline Sex & No. of Patients & Percentage & P value \\
\hline Male & 46 & $66 \%$ & \multirow{2}{*}{0.05} \\
\hline Female & 22 & $34 \%$ & \\
\hline Total & $\mathbf{6 8}$ & $\mathbf{1 0 0 \%}$ & \\
\hline \multicolumn{3}{|c|}{ Table 1. Sex Distribution } \\
\hline
\end{tabular}

Among the study group, 52 cases (76.4\%) presented as ulcer and 16 cases $(23.5 \%)$ as membrane like lesions.

In the study, the anatomical distribution of various ulcers and membranous lesions were buccal mucosa 41 cases (60.2\%), tongue 11 cases (16.1\%), lips 6 cases $(8.8 \%)$, gingiva 3 cases $(4.4 \%)$, hard palate 3 cases (4.4\%), anterior tonsillar pillar 2 cases $(2.9 \%)$ and soft palate 2 cases $(2.9 \%)$.

In our present study, patients having abuse/ habits were 45 cases $(66.1 \%)$. Various combined abuse/ habits found in these patients were smoking in 20 cases $(29.4 \%)$, alcohol in 18 cases (26.4\%), tobacco chewing in 23 cases (33.8\%), betel nuts in 22 cases (32.3\%), pan chewing in 20 cases (29.4\%) and gutka in 14 cases $(20.5 \%)$. In the study, duration of abuse/ habits were smoking for 25-30 yrs. of 27 cases (61.3\%), alcohol for 12 - 15 yrs. of 8 cases (18.1\%), Gutka chewing for 12 - 15 yrs. of 4 cases (9\%), tobacco chewing for 8 - 10 yrs. of 3 cases $(6.8 \%)$ and betel nuts and pan chewing for 8 - 10 yrs. of 3 cases $(6.8 \%)$.

Among the study group, 45 cases (66.1\%) had habits/ abuse and 23 cases (33.8\%) did not have any habits. Out of patients who had habits, 29 (42.6\%) were males and 16 $(23.5 \%)$ were females.

\begin{tabular}{|c|c|c|c|c|}
\hline Sl. No. & $\begin{array}{c}\text { Habits/ } \\
\text { Abuse }\end{array}$ & $\begin{array}{c}\text { No. of } \\
\text { Patients }\end{array}$ & $\begin{array}{c}\text { Per- } \\
\text { centage }\end{array}$ & P-value \\
\hline 1. & $\begin{array}{c}\text { Patients having } \\
\text { habits }\end{array}$ & 45 & $66.1 \%$ & \multirow{2}{*}{0.008} \\
\cline { 1 - 3 } 2. & $\begin{array}{c}\text { Patients not } \\
\text { having habits }\end{array}$ & 23 & $33.8 \%$ & \\
\cline { 1 - 3 } Table 2. Comparison with Patients having \\
Habits/Abuse and Patients not having Habits \\
\hline
\end{tabular}

Among the 10 cases of malignant lesions, 5 cases had abusive history of smoking, alcohol and tobacco chewing habits and 3 cases had betel nuts, pan chewing and gutka. Among 4 cases of leukoplakia, 1 had abusive history of smoking, alcohol and gutka chewing and 3 had history of betel nuts and pan chewing. In 12 cases of oral submucous fibrosis, 6 had abusive history of smoking and gutka chewing and 3 had history of alcohol, betel nuts and pan chewing.

In our study, various investigations have been done for 56 cases $(82.3 \%)$. Routine haematological tests were done. Out of these Random blood sugar 15 cases were elevated, HIV and HBsAg were positive in 3 cases, ESR levels were raised in 2 cases and 12 cases were anaemic with $\mathrm{Hb}<10 \mathrm{gm} \%$.

For 30 cases, Wedge biopsy was done and sent for histopathological examination. Out of these, 10 cases were diagnosed to have squamous cell carcinoma, 3 cases were pre-malignant lesions (leukoplakia), 4 cases were oral submucous fibrosis, 2 cases were lichen planus, 2 cases were pemphigus vulgaris and 9 cases were aphthous ulcers.

In our study, benign ulcers were 54 cases (79.4\%), premalignant lesions (leukoplakia) were 4 cases (5.8\%) and malignant lesions were 10 cases $(14.7 \%)$.

Among the study group various benign lesions were aphthous ulcers 25 cases (36.7\%), oral submucous fibrosis 12 cases $(17.6 \%)$, oral candidiasis 6 cases $(8.8 \%)$, dental ulcers 2 cases $(2.9 \%)$, pemphigus vulgaris 2 cases $(2.9 \%)$, traumatic ulcers 2 cases $(2.9 \%)$, lichen planus 2 cases $(2.9 \%)$ and follicular tonsillitis 3 cases (4.4\%).

The present study shows various lesions presented as ulcers and membranous lesions, among them patients came with ulcers were 23 cases of aphthous ulcers, 8 cases of oral submucous fibrosis, 10 cases of malignant lesions, 2 cases of dental ulcers, pemphigus vulgaris, traumatic ulcers, leukoplakia and lichen planus respectively. Cases presented as membrane like lesions were 2 cases of aphthous ulcers, 4 cases of oral submucous fibrosis, 6 cases of oral candidiasis, 3 of follicular tonsillitis and 2 of leukoplakia.

In our study, among various types of lesions 35 cases (51\%) were acute ulcers (less than 3 weeks), among them 20 were $(29.4 \%)$ aphthous ulcers (sporadic), 3 were $(4.4 \%)$ oral submucous fibrosis, 4 were $(5.8 \%)$ oral candidiasis, 2 were $(2.9 \%)$ leukoplakia and traumatic ulcers respectively, 1 was (1.4\%) dental ulcer, pemphigus vulgaris, lichen planus and follicular tonsillitis respectively. In chronic ulcers (more than 3 weeks) there were 33 cases (49\%), among them 5 were $(7.3 \%)$ aphthous ulcers (recurrent), 9 were $(13.2 \%)$ oral submucous fibrosis, 10 were $(14.7 \%)$ malignant lesions, 2 were $(2.9 \%)$ oral candidiasis, leukoplakia and follicular tonsillitis respectively, 1 was (1.4\%) dental ulcer, pemphigus vulgaris and lichen planus respectively.

\section{DISCUSSION}

- In our study, commonest benign ulcers constituting $80.8 \%(n=55)$ of all cases. Majority of them were Aphthous ulcers in buccal mucosa and lateral border of tongue. Other benign ulcers were oral submucous fibrosis $20.5 \%(n=14)$ and oral candidiasis $8.8 \%(\mathrm{n}=6)$. Next commonest benign ulcers were Dental ulcers $4.4 \%(n=3)$, Pemphigus vulgaris $4.4 \%(\mathrm{n}=3)$, Traumatic ulcers $2.9 \%(\mathrm{n}=2)$ and Lichen planus $2.9 \%(n=2)$.

- $\quad$ Pre-malignant ulcers like Leukoplakia were $4.4 \%$ $(n=3)$. Malignant ulcers were $14.7 \%(n=10)$. Peak age of presentation was $3^{\text {rd }}$ to $5^{\text {th }}$ decade, which was similar to study by Pheton JA et al. ${ }^{1}$

- Pheton JA 1991 and his colleagues have noted alcohol consumption and smoking are important aetiological factor in the malignant ulcers. ${ }^{1}$ 
- Traumatic ulcers and Lichen planus were rare representing less than $3 \%$ of non-malignant ulcers as reported by Pheton et al. ${ }^{1}$

- In our study, most common anatomical sites were buccal mucosa $60.2 \%(n=41)$ and lateral border of tongue $16.1 \%(n=11)$ comparable to the work of Pheton JA et al. ${ }^{1}$

- In the current study, ulcer and membrane like lesions were most common presenting symptoms similar to study by J. Phookan et al. ${ }^{2}$

- Oral submucous fibrosis was the second common benign ulcer observed in the study showing peak incidence in $2^{\text {nd }}$ to $4^{\text {th }}$ decade unlike study done by J. Phookan et al. ${ }^{2}$

- In our study, malignant ulcers $19.1 \% \quad(n=13)$ including 3 cases of Leukoplakia. This was comparable with study done by Priyanka Mahawar et $\mathrm{al}^{3}$ with $28 \%(\mathrm{n}=18)$. Squamous cell carcinoma was the most common malignancy observed in the study and constituted $14.7 \%$ of all malignant lesions similar to findings of Priyanka Mahawar et al. ${ }^{3}$

- A variety of oral mucosal lesions and conditions are associated with the habit of smoking and chewing tobacco, and many of these carry a potential risk for the development of cancer. ${ }^{4}$

- Lichen planus is a chronic inflammatory mucocutaneous disease, which frequently involves the oral mucosa. In many patients, the onset of OLP is insidious and patients are unaware of their oral condition. Some patients report a roughness of the lining of the mouth, sensitivity of the oral mucosa to hot or spicy foods, painful oral mucosa, red or white patches on the oral mucosa or oral ulcerations. ${ }^{5}$ In our case study, Traumatic ulcers were $2.9 \%(\mathrm{n}=2)$ and Lichen planus $2.9 \%(n=2)$ who were followed up for 6 months.

- In our study, most common benign ulcers were Aphthous ulcers, Oral submucous fibrosis and Oral candidiasis.

- $\quad$ Candidiasis is the most common oral opportunistic infection affecting people with HIV infection or AIDS. It is considered as an important marker of immune suppression and may be the initial manifestation of the disease in about $10 \%$ of HIVinfected adults. ${ }^{6}$

- Candida-associated denture stomatitis is the most common form of oral candidal infection with candida albicans being the principal aetiological agent. Candida adheres directly or via an intermediary layer of plaque-forming bacteria to denture acrylic. Despite antifungal therapy to treat denture stomatitis, infection is re-established soon after the treatment ceases. In addition, many predisposing factors have been identified as important in the development of oral candidiasis including malnourishment, common endocrine disorders such as diabetes mellitus, antibacterial drug therapy, corticosteroids, radiotherapy and other immunocompromised conditions. ${ }^{7}$

- Demonstration of fungi in biopsy specimens may require several serial sections to be cut. Fungi can be easily demonstrated and studied in tissue sections with special stains. The routinely used Haematoxylin and Eosin stain poorly stains Candida species. The specific fungal stains such as PAS stain, Grocott-Gomori's methenamine silver (GMS) and Gridley stains are widely used for demonstrating fungi in the tissues, which are coloured intensely with these stains. ${ }^{8}$

- Incidence of Oral candidiasis according to Isaac U et $\mathrm{al}^{9}$ is $0.4 \%$, but in our study Oral candidiasis is $8.8 \%$ $(n=6)$. Out of these, Acute ulcers (less than 3 weeks) were $5.8 \%(n=4)$ and Chronic ulcers were $2.9 \%$ $(\mathrm{n}=2)$.

- Peak age of occurrence of benign ulcers in our study was $2^{\text {nd }}, 3^{\text {rd }}, 4^{\text {th }}$ and 5 th decade $(80.8 \%)$, which was similar (56\%) to Isaac U and Isaac JS et al. ${ }^{9}$

- The catarrhal inflammation is a mild inflammation of mucous membrane. Stomatitis is a general term applied to inflammatory, erosive and ulcerative conditions widely affecting the mucous membranes which line the oral cavity. Gingivitis refers to inflammatory, erosive and ulcerative conditions which are confined to the mucoperiosteum covering the alveolar processes. ${ }^{10}$

- Raksha M 1983 noticed 6.1\% of the cases of cancers in India. In this study, it was determined $14.7 \%$ of the cases are malignant ulcers. ${ }^{11}$

- Pemphigus vulgaris (PV) is a chronic autoimmune mucocutaneous disease that usually manifests first in the oral cavity and may later spread to the skin or other mucous membranes. Apart from ulcers, vesicles, bullae and pustular lesions, it can present solely as mucosal erosions. ${ }^{12}$

- The recurrent aphthous ulcer (RAU) is a pathological change found in the oral mucosa, characterised by painful single or multiple ulcers. The aetiologic aspect of RAU is not well understood; however, it is known that due to lower CD4 cell counts patients had higher prevalence of these oral lesions and immunosuppressed patients with HIV are predisposed. ${ }^{13}$

\section{CONCLUSION}

In our study, we concluded that most common oral cavity lesions were of benign nature. However, the origin and nature of the oral cavity lesions cannot be confirmed by clinical examination alone; hence, it is advisable to have a histopathological examination to confirm the nature of the lesion. This is important as treatment strategies must be directed towards providing symptomatic relief by reducing pain, increase in duration of ulcer-free periods and accelerating ulcer healing. This is true in cases of most benign lesions including aphthous ulcer.

We observed marked presence of main risk factors like tobacco chewing, chewing of betel quid and with and/or without tobacco and gutka chewing, not only for oral cancer and pre-cancer but also for many other oral lesions such as lichen planus, leukoplakia, lichenoid lesion and smoker's palate.

In our study, most of the patients were in the age group between $3^{\text {rd }}$, $4^{\text {th }}$ and $5^{\text {th }}$ decade with above mentioned habits. We recommend those aged more than or equal to $40 \mathrm{yrs}$. 
undergo oral mucosa screening regularly, so that potential oral cancer can be identified as early as possible. We observed equal occurrence of malignancy in patients above and below 40 yrs. So we recommend oral screening for all who have the above mentioned habits.

The prevalence of oral pre-malignant lesions was quite high in this part of country. Buccal mucosa was found to be most common site of involvement. All the cases were found to be associated with betel nut chewing habit. Hence, screening is the key.

The risk of oral submucous fibrosis associated with gutka chewing habit and leukoplakia, erythroplakia and oral cancer associated with smoking, chewing or mixed habits are quite high. Therefore, intervention programs to discourage the use of gutka and other risky habits should be a public health priority.

Oral submucous fibrosis is a crippling disease of the oral cavity, which is poorly understood and unsatisfactorily treated oral lesions because of its multifactorial aetiology. No single drug has provided a complete relief and this is mainly due to the fact that the aetiology of the disease is not fully understood. The better approach in demystifying the pathogenesis of OSF could provide a new and successful therapeutic direction in near future.

Oral lesions serve as early marker for HIV infection and may herald deterioration in general health and a poor prognosis. We must be well aware of the characteristics and presentation of the manifestations of HIV infection, thus enabling early identification of HIV and ensuring timely initiation of therapy.

A candidal infection is the first clinical sign of HIV infection, presence of oral candidiasis without a local cause such as xerostomia or therapy with antimicrobials, corticosteroids or other immune suppressive drugs in a person who otherwise appears healthy should prompt investigation into lifestyle and other factors pertaining to the risk of HIV infection. The HIV-related oral lesions are hence regarded as "sentinels and signposts" of HIV/AIDS and their early recognition and prompt management are of paramount importance in maintaining the health and prolonging the life of patients with AIDS.

Early diagnosis of oral cancer is a priority health objective, in which oral health professionals may play a pivotal role. Detection should lead to less damage from cancer therapy and to a better prognosis. Scalpel biopsy and wedge biopsy are effective diagnostic tests for evaluating suspicious oral lesions, which may be precancerous or cancerous. Light based screening aids should only be employed as an adjunct to the clinical examination for identifying oral lesions that may have been overlooked with a conventional oral examination. However, controlled trials in both high and low risk populations with histologic outcomes and critical appraisal from the medical community are required before they can be integrated into practice.

\section{REFERENCES}

[1] Phelan JA, Eisig S, Freedman PD, et al. Major aphthous like ulcers in patients with AIDS. Oral Surg Oral Med Oral Pathol 1991;71(1):68-72.

[2] Phookan J, Saikia KP. Clinicopathological study of the pre-malignant conditions of oral cavity. IJO \& HNS 1998;50(3):246-9.

[3] Mahawar P, Anand S, Sinha U, et al. Screening for premalignant conditions in the oral cavity of chronic tobacco chewers. National Journal of Community Medicine 2011;2(1):82-5.

[4] Boorghani M, Gholizadeh N, Zenouz AT, et al. Oral lichen planus: clinical features, etiology, treatment and management: a review of literature. Journal of Dent Res Dent Clin Dent Prospects 2010;4(1):3-9.

[5] Patil PB, Bathi R, Chaudhari S. Prevalence of oral mucosal lesions in dental patients with tobacco smoking, chewing, and mixed habits: a cross-sectional study in South India. J Family Community Med 2013;20(2):130-5.

[6] Shetti A, Gupta I, Charantimath SM. Oral candidiasis: aiding in the diagnosis of HIV-a case report. Case Reports in Dentistry Article ID 929616, 2011;2011: p. 4.

[7] Anibal PC, de Cassia OSJ, Peixoto ITA, et al. Conventional and alternative antifungal therapies to oral candidiasis. Brazilian Journal of Microbiology 2010;41(4):824-31.

[8] Preeti L, Magesh KT, Rajkumar K, et al. Recurrent aphthous stomatitis. Indian Journal of Pathology 2011:23-8.

[9] Isaac U, Issac JS, Khoso NA. Histopathologic features of oral submucous fibrosis: a study of 35 biopsy specimens. Oral Surg Oral Med Oral Pathol Oral Radiol Endod 2008;106(4):556-60.

[10] Antoon JW, Miller RL. Aphthous ulcers--a review of the literature on aetiology, pathogenesis, diagnosis and treatment. J American Dental Association 1980;101(5):803-8.

[11] Shah RM. Oral pemphigus vulgaris clinic-pathological follow-up of 34 cases. J Oral Medicine 1983;38:170-3.

[12] Javali MR, Zainab H. Pemphigus Vulgaris as gingival involvement. Indian Dermatological Online Journal 2012;3(3):202-4.

[13] Caputo BV, Filho NGA, Dos Santos CC, et al. Laser therapy of recurrent aphthous ulcer in patients with HIV infections. Case Rep Med Article ID 695642, 2012;2012: p. 3. 